\title{
FAS engagement drives apoptosis of enterocytes of coeliac patients
}

\author{
L Maiuri, C Ciacci, V Raia, L Vacca, I Ricciardelli, F Raimondi, S Auricchio, S Quaratino, \\ $M$ Londei
}

\begin{abstract}
Background-Villus atrophy is the most distinctive sign of untreated coeliac disease (CD) and epithelial apoptosis is considered to be involved in this stage of the coeliac lesion. The extent of villus atrophy is, however, not homogeneous and patients with patchy or mild lesions have been described.
\end{abstract}

Aims-To address: (a) the degree of "patchiness" in untreated CD patients; and (b) to clarify if apoptosis, and eventually which trigger drives it, causes epithelial damage.

Patients-Twenty of 40 untreated, 14 treated coeliac patients, and 15 controls received five or more multiple duodenal biopsies; the remaining 20 untreated CD patients had no more than three biopsies. Methods-All biopsies were analysed to monitor the presence of a "flat" mucosa. Biopsies of 14 untreated, 10 treated coeliacs, and seven controls were cultured with or without gliadin. DNA fragmentation was studied by terminal deoxynucleotidyl transferase (TdT) mediated dUTP digoxigenin nick end labelling (TUNEL), and FAS and Ki67 expression by immunohistochemistry. Antiendomysium antibodies (EMA) were surveyed in biopsy culture supernatants.

Results-A pattern of patchy duodenal lesions was observed in all untreated CD patients biopsied up to five times. High enterocyte FAS expression, and a high number of TUNEL+ and $\mathrm{Ki67+}$ enterocytes were detected in areas with villus atrophy but not in those with a normal morphology $(p<0.001)$. Conversely, EMA in culture supernatants and signs of immunological activation were present in all untreated CD biopsies. In vitro gliadin challenge increased the number of TUNEL+ and Ki67+ enterocytes $(p<0.001$ $v$ cultures with medium alone) only in "flat" biopsies. Neutralising anti-FAS monoclonal antibodies were found to control gliadin induced enterocyte apoptosis (p>0.01) while agonist anti-FAS monoclonal antibody increased it $(p<0.001)$. Conclusions-Patchy lesions are observed in untreated CD mucosa and epithelial FAS engagement is a key trigger in driving villus atrophy in CD.

(Gut 2001;48:418-424)

Keywords: apoptosis; FAS; enterocytes; coeliac disease
Coeliac disease (CD) is a frequent and, to date, under diagnosed disease of the small intestine characterised by the presence of endomysium antibodies (EMA) in the sera of untreated CD patients. ${ }^{1-3}$ Three of the major histological features of the small intestinal mucosa of untreated CD patients are: gliadin dependent activation of supposed pathogenic $\mathrm{T}$ cells; $\mathrm{T}$ lymphocyte intraepithelial infiltration; and crypt hyperplasia with villus atrophy. ${ }^{2}$ The latter, the "flat" mucosa, is possibly the most canonical feature of CD although it cannot be considered in isolation but in the wider context of a remodelling process of the small intestine. ${ }^{1-4}$ It is now accepted that CD is not limited to patients with severe enteropathy but can also be applied to patients with minor pathological changes, where "patchy" lesions are sometimes observed. ${ }^{56}$ Evidence of patchy duodenal lesions in the same CD patient provided an intriguing descriptive study of mucosal damage in CD but an explanation for these unexpected findings is still awaited. Here, we analysed a larger sample of CD patients by multiple endoscopic biopsies to fully evaluate the extent of the patchy lesion profile and also to unravel the mysteries controlling induction of the flat mucosa.

It has previously been reported that DNA fragmentation is observed in the majority of enterocytes of untreated CD intestine and that after gluten withdrawal, a normal profile is restored. ${ }^{7}$ One of the most studied pathways leading to apoptosis is by FAS engagement, the prototype of the "death" receptors widely expressed by many cell types ${ }^{8}$ which we have recently reported to be rapidly upregulated in treated coeliac duodenum on in vitro challenge with gliadin. ${ }^{9}$

Analysis of patchy lesions in coeliac duodenum represents an ideal tool to clarify the correlation among FAS expression, enterocyte apoptosis, crypt epithelial proliferation, and villus atrophy. Moreover, using the organ culture model of coeliac duodenal explants, ${ }^{9-13}$ we tested the true role of FAS engagement in driving epithelial apoptosis and villus flattening in CD using agonist or antagonist anti-FAS monoclonal antibodies (mAbs).

Our study indicates that a definite correlation between villus flattening, enterocyte FAS expression, apoptosis, and crypt cell prolifera-

Abbreviations used in this paper: $\mathrm{CD}$, coeliac disease; EMA, endomysium antibodies; mAb, monoclonal antibody; Ig, immunoglobulin; PT, peptic-tryptic; TUNEL, terminal deoxynucleotidyl transferase (TdT) mediated dUTP digoxigenin nick end labelling; IEL, intraepithelial lymphocytes. 
tion is present in coeliac duodenum. Moreover, our data clearly suggest that FAS engagement plays a key role in induction of epithelial damage and that the difference between patchy lesions and a normal histology is the specific lack of expression of FAS. Hence our studies may help in the understanding of the precise correlation that exists between induction of damage and the remodelling process observed in CD.

\section{Methods}

PATIENTS

Forty untreated CD patients (mean age 31.2, range 3-54) underwent duodenal endoscopy and biopsy for diagnostic purposes. In all patients serum anti-EMA were detected. Twenty patients underwent less than three biopsies which all revealed villus atrophy on routine histological examination. The other 20 patients underwent at least five biopsies, examined immediately at the dissecting microscope. In 12 of these 20 patients the first sample analysed showed villus flattening whereas in eight patients the first sample showed apparent normal villi. Analysis at the dissecting microscope of all fragments obtained by multiple endoscopic biopsies revealed a patchy duodenal lesion with various extension of the villus atrophy along the duodenum in all 20 patients. For each patient, one fragment with villus flattening and one sample with apparent normal villi were embedded in OCT and used for histological analysis to confirm the appearance observed at the dissecting microscope. The other fragments (with villus atrophy or normal villi) were divided into two parts each and cultured as described below.

Fourteen of the untreated patients (mean age (range) 37.3 (21-53) years) were studied after a strict gluten free diet spanning a period of at least 12 months when they were rebiopsied (termed treated CD patients). All were EMA negative at the time of the second biopsy.

Fifteen non-CD patients (mean age (range) 31.1 (14-60) years), undergoing duodenal endoscopy because of oesophagitis $(n=5)$, gastritis $(n=5)$, or chronic non-specific diarrhoea $(n=5)$ had multiple biopsies. In all biopsies, all fragments showed normal villus architecture at the dissecting microscope as well as after histological analysis. Informed consent was obtained from all CD patients and controls.

IN VITRO ORGAN CULTURE STUDIES

Duodenal explants from duodenal regions with villus atrophy from 10 untreated CD patients were cultured in vitro ${ }^{9-11}$ in the presence or absence of a peptic-tryptic (PT) gliadin digest $(1 \mathrm{mg} / \mathrm{ml})$ with or without neutralising antiFAS (M3) mAb (in seven cases) (Immunex Corp, Seattle, USA, $5 \mu \mathrm{g} / \mathrm{ml}),{ }^{14}$ and/or with maize digest $(1 \mathrm{mg} / \mathrm{ml})$ (in three cases). In vitro cultures with medium together with agonist anti-FAS CH-11 mAb (Beckman-Coulter Inc., Brea, California, USA) were also performed in four cases: anti-FAS CH-11 mAb was used in a range of concentrations ( $100 \mathrm{ng}$ to $5 \mu \mathrm{g} / \mathrm{ml}$ ) as this antibody has never been used under these experimental conditions. Neutralising antiFAS M38 mAb was also tested in three cultures and the results correlated with those obtained using neutralising anti-FAS M3 mAb. Control antibodies anti-CD80 (5 $\mu \mathrm{g} / \mathrm{ml}$ ) (in four cases) or anti-human lactase $\mathrm{mAb}$ mlac1 $(5 \mu \mathrm{g} / \mathrm{ml})^{15}$ (in four cases) as well as CTLA4immunoglobulin (Ig) (Genetic Institute Corporation, Cambridge, USA) (in five cases) ${ }^{9}$ were also added to gliadin.

Duodenal explants from duodenal regions with normal villus architecture from four untreated CD patients were cultured in the presence or absence of a PT gliadin digest (1 $\mathrm{mg} / \mathrm{ml}$ ) or agonist anti-FAS CH-11 mAb.

Duodenal explants from 10 treated CD patients were cultured in vitro for 24 hours $^{9-11}$ in the presence of culture medium with or without a PT digest of gliadin $(1 \mathrm{mg} / \mathrm{ml})$ (in all cases), and with maize prolamin peptides (1 $\mathrm{mg} / \mathrm{ml}$ ) (in six cases) or agonist anti-FAS $\mathrm{CH}-11 \mathrm{mAb}$ (in four cases). In seven controls, in vitro cultures with or without PT gliadin digest $(1 \mathrm{mg} / \mathrm{ml})$ were performed; in three cases agonist anti-FAS CH-11 mAb was also tested.

DETECTION OF DNA FRAGMENTATION

DNA fragmentation on tissue sections was assayed in all patients as previously reported ${ }^{16}$ by terminal deoxynucleotidyl transferase (TdT) mediated dUTP digoxigenin nick end labelling (TUNEL). In CD subjects with patchy lesions, DNA fragmentation was assessed in samples with villus atrophy as well as in those with a normal mucosal architecture. Specificity control experiments were performed as previously reported. ${ }^{16}$

Two colour immunofluorescence was used to determine whether apoptotic cells were $\mathrm{T}$ cells or epithelial cells. The experiments were carried out by developing the TUNEL reaction with phycoeritrin (RPE) conjugated antidigoxigenin mAb (Boehringer, Mannheim, Germany) and simultaneously incubating tissue sections with anti-CD3 mAb (1:100; Dako, Copenhagen, Denmark) followed by FITC conjugated antimouse Ig (1:30; Dako).

IMMUNOHISTOCHEMISTRY

Antigen detection on frozen tissue sections was performed by immunohistochemistry as previously reported ${ }^{5}{ }^{10}$ with mAbs anti-Ki67 (1:25; Dako) or CD3 (1:100; Dako), anti-FAS (M3 and M38) (mouse IgG1, 1:30; Immunex Corp), CD25 (1:30; Dako), and ICAM1 (1:400; 509 Ylem CVA) by peroxidase or alkaline phosphatase staining techniques according to a method previously described. ${ }^{9}$ Anti-FASligand $\mathrm{mAbs}$ (A11 and $\mathrm{H} 11$ rat biotinylated $\mathrm{Ig}$ 1:60; Alexis-Corporation, San Diego, California, USA) were used to detect FAS-ligand expression in biopsies from CD patients. The antibodies were visualised using a peroxidase staining technique following by a method previously described. ${ }^{9}$ Numbers of intraepithelial lymphocytes (IEL) were counted as CD3+ cells $/ 100$ enterocytes $^{10}$ and Ki67+ enterocytes as percentage of crypt enterocytes. Staining of epithelial cells by anti-FAS mAb was arbitrarily graded from 0 to +2 , based on the intensity of 
Table 1 Overall representation of the characteristics of the mucosal manifestations in coeliac disease (CD) and controls

\begin{tabular}{lllll}
\hline & \multicolumn{2}{l}{ Untreated CD } & & \\
\cline { 2 - 3 } & Villus atrophy & Normal mucosa & \multirow{2}{*}{ Treated CD } & \multirow{2}{*}{ Controls } \\
\hline Enterocyte apoptosis & High & Low & Low & Low \\
Enterocyte Ki67 expression & High & Low & Low & Low \\
Enterocyte FAS expression & High & Low & Low & Low \\
IEL FAS-ligand expression & High & High & Low & Low \\
LPMNC activation & High & High & Low & Low \\
EMA in culture supernatant & Present & Present & Absent & Absent \\
& & & &
\end{tabular}

staining in more than $70 \%$ of cells (undetectable $=0$, low $=1+$, intense $=2+$ ). Guidelines for the scoring system were established at the start of the study and four observers independently analysed the samples; the results were compared afterwards.

At least five slides for each sample were blindly evaluated. Specificity control experiments were performed using mouse IgG or IgM against inappropriate blood group antigens, and simultaneously analysing different cultured samples belonging to the same individual.

DETECTION OF EMA IN CULTURE SUPERNATANTS EMA were detected in culture supernatants of cultured untreated CD biopsies (10 from areas with villus atrophy and four from areas with
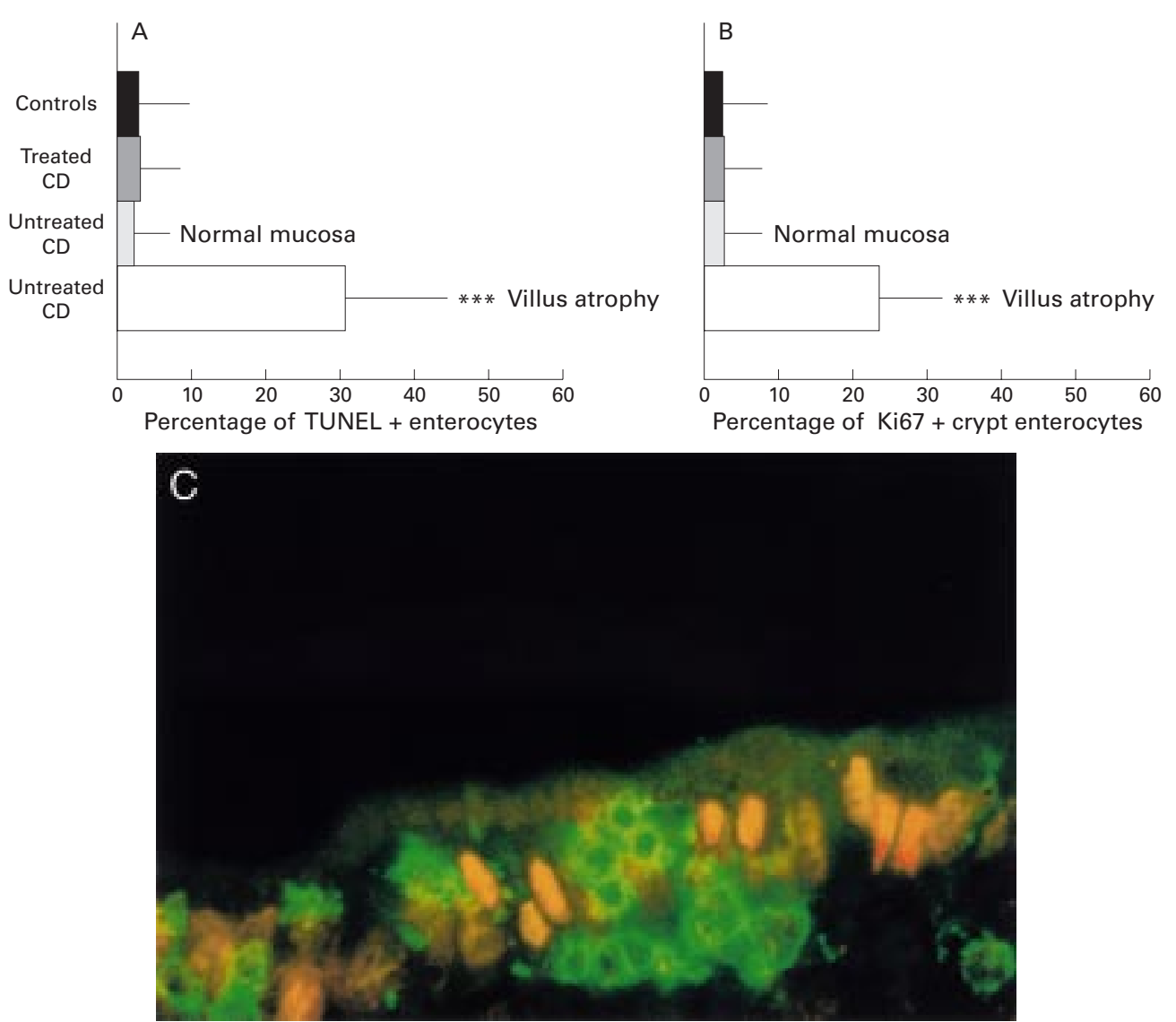

Figure 1 DNA fragmentation and Ki67 expression in coeliac disease (CD) and control intestine. (A) DNA fragmentation in enterocytes of $C D$ and control intestine. (B) Expression of Ki67 in crypt enterocytes of $C D$ and control intestine (mean (SD)). Untreated $C D$, untreated biopsies with patchy mucosal lesions $(n=20)$; treated $C D$, treated biopsies $(n=14)$; controls, biopsies from non-coeliac patients with a normal villus architecture $(n=15) .{ }^{\star * *} p<0.0001 v$ treated $C D$ and controls. (C) Pattern of intraepithelial DNA fragmentation in untreated CD duodenum. DNA fragmentation (TUNEL technique, red colour) is absent in the nucleus of all intraepithelial lymphocytes (CD3, green colour), while it is detected in the majority of enterocytes. Original magnification $\times 240$. Two colour immunofluorescence: TUNEL technique, RPE labelling (red); immunohistochemistry, CD3 labelling (green) (see methods).

normal histology) after incubation with medium alone, in accordance with methods STATISTICAL ANALYSIS

Samples from each category were compared. The Student's $t$ test for paired samples was used to discriminate between untreated CD enterocytes after challenge with medium alone, with medium supplemented with gliadin, or with gliadin supplemented with mAbs. Nonparametric tests (Wilcoxon test) were also obtained using parametric tests. Fisher's test was used to compare tissues with undetectable or low FAS expression (0 to $1+)$ with those was used to compare FAS expression in tissue specimens after gliadin challenge with those after challenge with medium alone.

PATCHY MUCOSAL LESIONS ARE FREQUENTLY OBSERVED IN UNTREATED CD DUODENUM In all samples, duodenal mucosal findings tained using the dissecting microscope were confirmed by histological analysis. In the 20 untreated CD biopsies in which more than five multiple biopsies were performed, we observed 


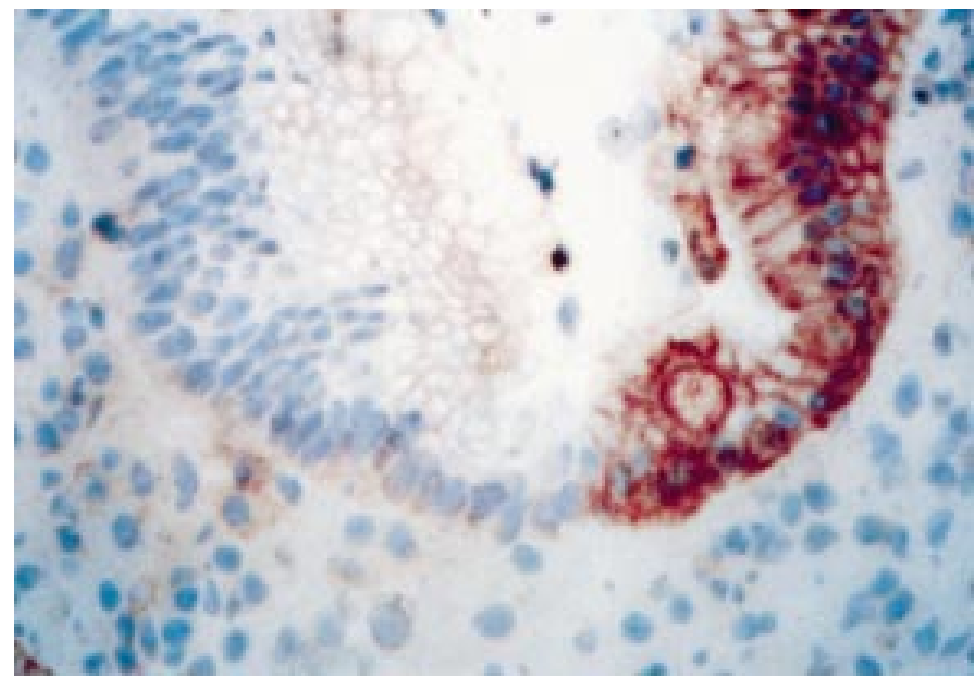

Figure 2 FAS expression in untreated coeliac disease (CD) duodenum. A marked change in expression of FAS is observed at the border between regions with normal villus architecture (low expression; left side) and those with villus atrophy (very high expression; right side). Original magnification $\times 150$; immunohistochemistry, peroxidase staining technique.

that the flat area corresponded in all cases to villus atrophy and crypt hyperplasia, and in 18/20 samples increased IEL counts (above 40/100 enterocytes) were detected. Well represented villi observed with the dissecting microscope corresponded to normal villus length with a villus height/crypt depth ratio higher than $3,{ }^{17}$ and IEL counts greater than $40 / 100$ enterocytes were found in only $13 / 20$ samples. In all untreated $\mathrm{CD}$ samples, regardless of mucosal damage, common features of immunological activation ${ }^{9}{ }^{10}$ were found in the lamina propria (number of $\mathrm{CD} 25+$ and ICAM1+ cells in the lamina propria higher than mean +3 SD of control values), as previously reported by us ${ }^{5}$ (table 1 ).

DNA FRAGMENTATION, AND Ki67 AND FAS EXPRESSION IN ENTEROCYTES CORRELATE WITH MUCOSAL LESIONS

Untreated CD samples with villus atrophy In untreated CD biopsy samples with villus atrophy, TUNEL positivity was increased compared with controls $(\mathrm{p}<0.0001)$ (fig 1A) and expression of $\mathrm{Ki} 67$ was strongly augmented in crypts, in agreement with previous reports $^{37} \quad(\mathrm{p}<0.0001 v$ controls) (fig 1B). Virtually no CD3+ cells stained TUNEL positive, indicating that all apoptotic cells were epithelial. Interestingly, some TUNEL positive (red) epithelial cells were not near to CD3+ (green) (fig 1C). This finding may indicate that not all epithelial death is caused by infiltrating FAS-ligand positive IEL and that other mechanisms may be involved, although no definitive conclusions can be drawn at present. Intense FAS expression $(2+)$ in surface and crypt enterocytes was found in $16 / 20$ samples. In these samples expression of FAS was detected in the majority (more than $70 \%$ ) of enterocytes ( $v$ $0 / 15$ in controls; $\mathrm{p}<0.01$ ) (table 1 ).

FAS-ligand expression was detected in IEL (more than $50 \%$ ) of all but four untreated CD biopsies with villus atrophy (data not shown). Interestingly, the pattern of FAS-ligand expres- sion was highly reminiscent of that described by Viard et al in patients with Lyell's syndrome. ${ }^{18}$

Untreated CD samples with normal morphology In all untreated $C D$ samples with a normal mucosal architecture, the pattern of DNA fragmentation (fig 1A) and Ki67 antigen expression (fig $1 \mathrm{~B})$ was similar to controls $(\mathrm{p}>0.5)$. Also, high FAS expression was not found in any of the eight cases ( $p>0.5 v$ controls). In a few cases we detected a sharp change in expression of FAS at the border between flat (high) and normal (low) areas (fig 2).

FAS-ligand expression in untreated CD intestine with normal villus architecture was similar to that found in untreated CD with villus atrophy (table 1).

Treated CD samples

In all treated CD samples the pattern of DNA fragmentation (fig 1A) and Ki67 antigen expression (fig 1B) was similar to that observed in controls $(p>0.5)$. High enterocyte FAS expression was observed only in $1 / 14$ cases ( $p>0.5 v$ controls). In these samples no evidence of immunological activation was observed (table 1), as previously reported..$^{5}$ IEL in treated CD and controls were virtually all FAS-ligand negative (table 1).

\section{ORGAN CULTURE STUDIES}

In untreated CD biopsies with villus atrophy, gliadin induces an increase in enterocyte apoptosis via a FAS dependent pathway

Increased expression of FAS in enterocytes did not automatically mean that FAS engagement was crucial in driving epithelial apoptosis. We used in vitro culture studies to verify whether or not expressed FAS could have delivered a functional signal.

In seven cultured untreated CD samples with villus atrophy in which FAS expression was intense before in vitro manipulation, 24 hour gliadin challenge caused an increase in the number of TUNEL positive enterocytes compared with the profile observed with medium alone $(\mathrm{p}<0.001)$ (fig 3A). Expression of Ki67 was markedly decreased after incubation with medium alone $(\mathrm{p}<0.0001)$ whereas gliadin sustained Ki67 expression observed before in vitro manipulation ( $p>0.5$ ) (fig $3 B$ ).

Both neutralising anti-FAS antibodies were effective in reducing the number of TUNEL+ enterocytes compared with the profile observed in vitro after incubation with gliadin alone $(p<0.001)$ (fig 3A, C, D) while they failed to control Ki67 expression (data not shown). No protective effect was observed after incubation with anti-human lactase $\operatorname{mlac} 1^{15}$ or anti-CD80 mAbs, which bear the same isotype of the neutralising anti-FAS antibody in four tested cases $(p>0.5)$ (fig $3 A$ ). In five of the cultured untreated CD biopsies with villus atrophy, CTLA4-Ig, a powerful and specific inhibitor of T cell activation, ${ }^{19}$ was unable to control the gliadin induced increase in DNA fragmentation of enterocytes $(\mathrm{p}>0.5)$ (fig $3 \mathrm{~A})$. However, CTLA-4Ig downregulated the pattern of mucosal immune activation (data not shown) indi- 

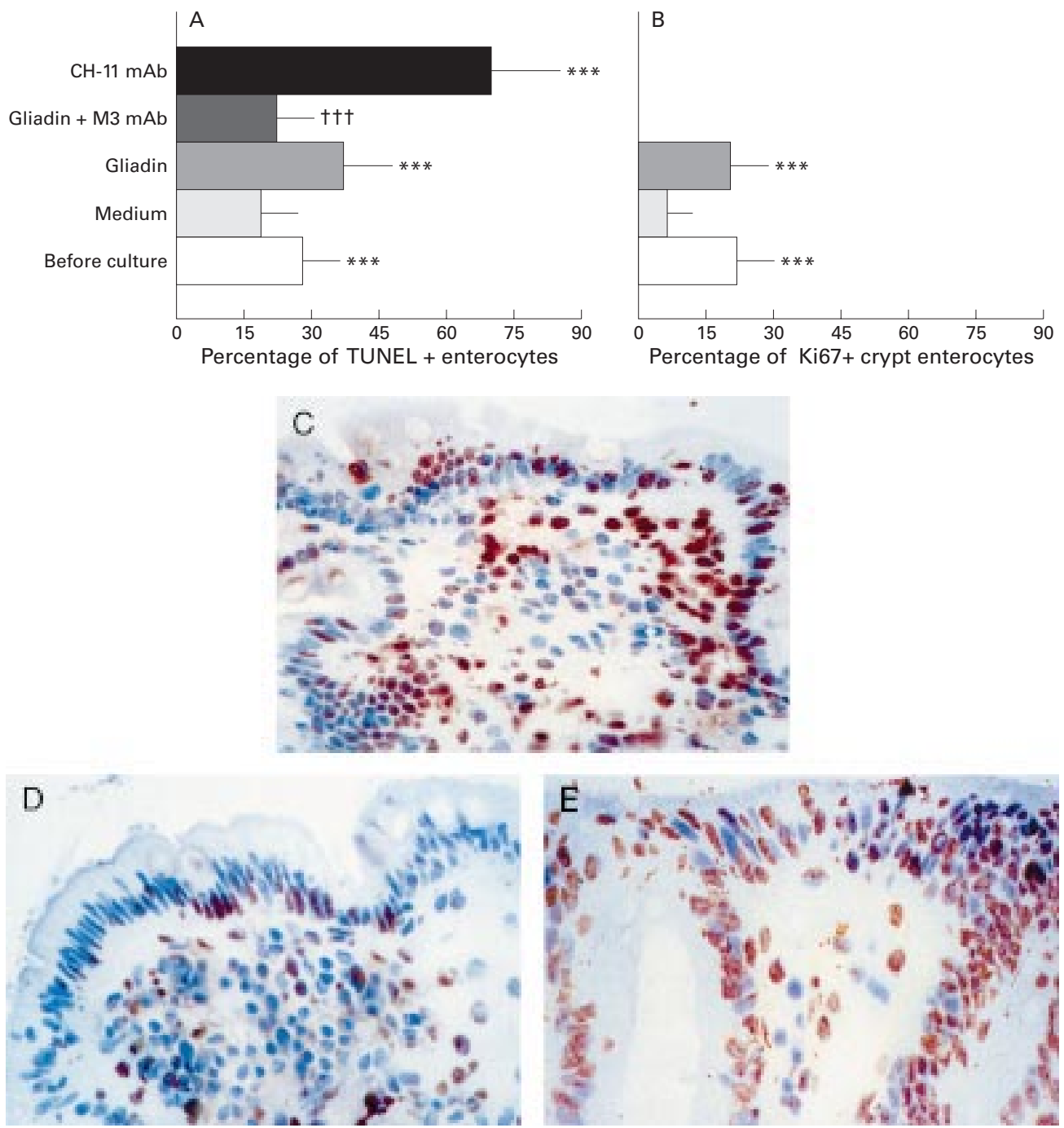

Figure 3 In vitro organ culture of untreated coeliac disease (CD) intestine with villus atrophy: DNA fragmentation and Ki67 expression after 24 hour challenge with medium or gliadin. Effect of neutralising anti-FAS M3 or agonist anti-FAS CH-11 monoclonal antibodies (mAbs) on enterocyte apoptosis. (A) DNA fragmentation and (B) Ki67 expression before and after in vitro culture. Untreated $C D$ samples with villus atrophy before in vitro culture $(n=7)$ and after 24 hour challenge with medium alone $(n=7)$, gliadin $(n=7)$, gliadin supplemented with anti-FAS M3 $m A b(n=7)$, or medium supplemented with anti-FAS CH-11 $\mathrm{mAb}(n=4)$. The number of TUNEL+ enterocytes (mean (SD)) are given as percentage of both surface and crypt enterocytes and Ki67+ enterocytes (mean (SD)) as percentage of crypt enterocytes. $\star \star * ⿻ 00.001 \mathrm{v}$ samples after 24 hour challenge with medium alone; $t+t p=0.001 \mathrm{v}$ samples after 24 hour gliadin challenge. (C) Pattern of DNA fragmentation after 24 hour gliadin challenge. Note that the majority of enterocytes are TUNEL+; staining is also evident in many lamina propria mononuclear cells. (D) Pattern of DNA fragmentation after 24 hours of in vitro challenge with gliadin supplemented with neutralising anti-FAS M3 mAb. The number of TUNEL+ enterocytes is decreased compared with the pattern observed after gliadin challenge. (E) Pattern of DNA fragmentation after 24 hours of in vitro challenge with medium supplemented with agonist $\mathrm{CH}-11$ anti-FAS $\mathrm{mAb}$. A marked increase in the number of TUNEL+ enterocytes with derangement of epithelial architecture is evident. Original magnification: $C, D$ and $E(\times 180)$; TUNEL technique, peroxidase staining.

cating its efficacy in controlling the immunomediated gliadin induced activation. In four cultured untreated CD samples with villus atrophy, agonist $\mathrm{CH}-11$ anti-FAS mAbs induced a dramatic increase in TUNEL+ enterocytes and derangement of the mucosal architecture $(\mathrm{p}<0.001)$ (fig 3A, E) while it did not increase Ki67 expression (data not shown) (table 2).

In untreated $C D$ biopsies with normal villus architecture, low enterocyte FAS expression was unable to launch the apoptotic programme In the four cultured untreated CD samples with normal villus architecture in which FAS expression was low to absent before in vitro culture, triggering of the FAS receptor with agonist $\mathrm{CH}-11$ anti-FAS mAbs was not effective in inducing enterocyte apoptosis ( $\mathrm{p}>0.5 v$ cultures with medium alone) (fig $4 \mathrm{~A}$ ). A 24 hour gliadin challenge was similarly unable to increase the number of TUNEL+ enterocytes (fig 4A) or to increase $\mathrm{Ki67}$ expression in crypts (fig 4B) ( $p>0.5 v$ cultures with medium alone). Moreover, in these biopsies, no increased expression of enterocyte FAS receptor was induced by gliadin challenge in any of the four cultured biopsies ( $\mathrm{p}>0.5 v$ cultures with medium alone) (table 2).

Untreated CD biopsies release EMA in culture supernatants regardless of duodenal morphology In all cultured untreated CD samples with villus atrophy, analysis of culture supernatants ${ }^{11}$ revealed spontaneous release of EMA in the 
Table 2 Overall representation of the characteristics of the mucosal manifestations in organ culture of the small intestine of coeliac disease (CD) patients and controls

\begin{tabular}{|c|c|c|c|c|}
\hline & \multicolumn{2}{|l|}{ Untreated $C D$} & \multirow[b]{2}{*}{ Treated $C D$} & \multirow[b]{2}{*}{ Controls } \\
\hline & Villus atrophy & Normal mucosa & & \\
\hline Increase in enterocyte apoptosis ${ }^{\star}$ & Yes & No & No & No \\
\hline $\begin{array}{l}\text { Increase in enterocyte Ki67 } \\
\text { expression }{ }^{\star}\end{array}$ & Yes & No & No & No \\
\hline $\begin{array}{l}\text { Increase in enterocyte FAS } \\
\text { expression }\end{array}$ & Yes & No & Yes & No \\
\hline
\end{tabular}

${ }^{\star}$ Organ cultures with gliadin digest $v$ cultures with medium alone.

absence of gliadin stimulation, in accordance with previous reports. ${ }^{11}{ }^{20}$ Interestingly, spontaneous release of EMA was detected in culture supernatants even in all four cultured untreated biopsies with normal mucosal architecture (table 2).

In treated $C D$ samples, gliadin induces FAS expression in enterocytes

In all 10 cultured treated CD samples, 24 hour gliadin challenge was unable to increase the number of TUNEL+ or Ki67+ enterocytes (data not shown) while it was effective in enhancing enterocyte FAS expression in $7 / 10$ cases $(\mathrm{p}<0.01)$, as described in a previous report $^{9}$ (fig 5A-5C) (table 2).

\section{Discussion}

Complex patterns of events control the evolution of the mucosal manifestations of $\mathrm{CD}$, which is characterised by clear signs of mucosal damage and concomitant features of tissue remodelling. ${ }^{1-4}$ The final result of this destructive/remodelling activity is believed to be an evenly distributed "flat" mucosa with an overall increase in the whole mucosa with crypt enlargement. ${ }^{2}$ This pathological pattern is customarily believed to be caused by immunological recognition of gliadin. ${ }^{21}$ However, it is recognised that patchy lesions are observed in some coeliac patients ${ }^{5}{ }^{6}$ but the proportion with a patchy pattern is not clearly defined and is considered to be infrequent. This study provides several significant findings and clarifies some of the pathogenic features of CD. In the first instance, we observed that patchy lesions are frequently observed in untreated CD mucosa and that they are not the exception in untreated CD duodenum. This may have an important clinical significance as diagnosis of CD is still widely based on small intestine histology. ${ }^{2}$ We found that patchiness was not rare and conversely quite frequent in untreated CD, and hence some patients could be wrongly diagnosed as not having CD.
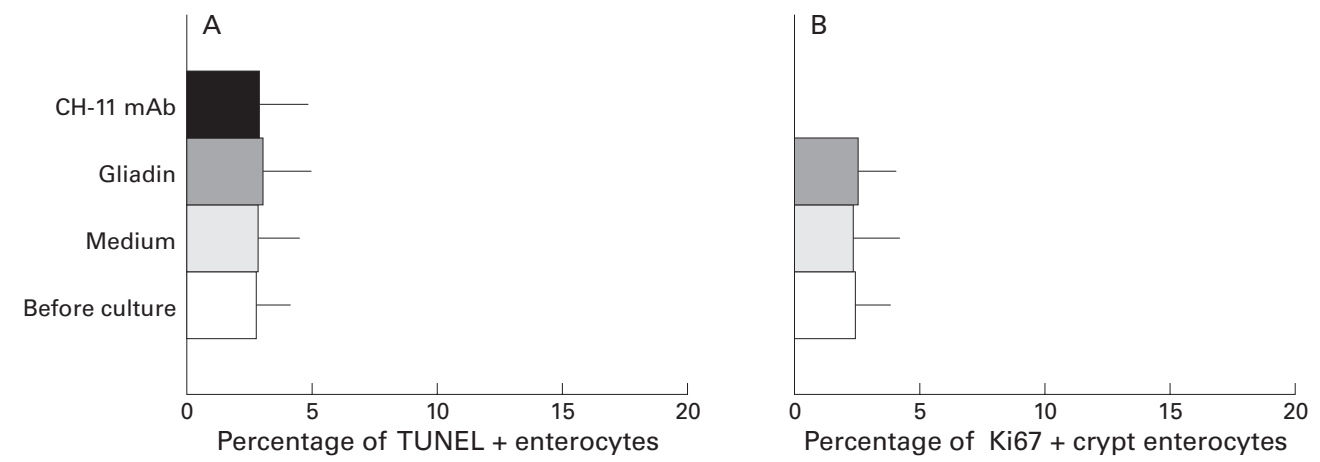

Figure 4 In vitro organ culture of untreated coeliac disease (CD) intestine with normal villus architecture. DNA fragmentation (A) and Ki67 expression (B) (mean (SD)) after 24 hour challenge with medium or gliadin and effect of agonist anti-FAS CH-11 $\mathrm{mAb}$ on enterocyte apoptosis
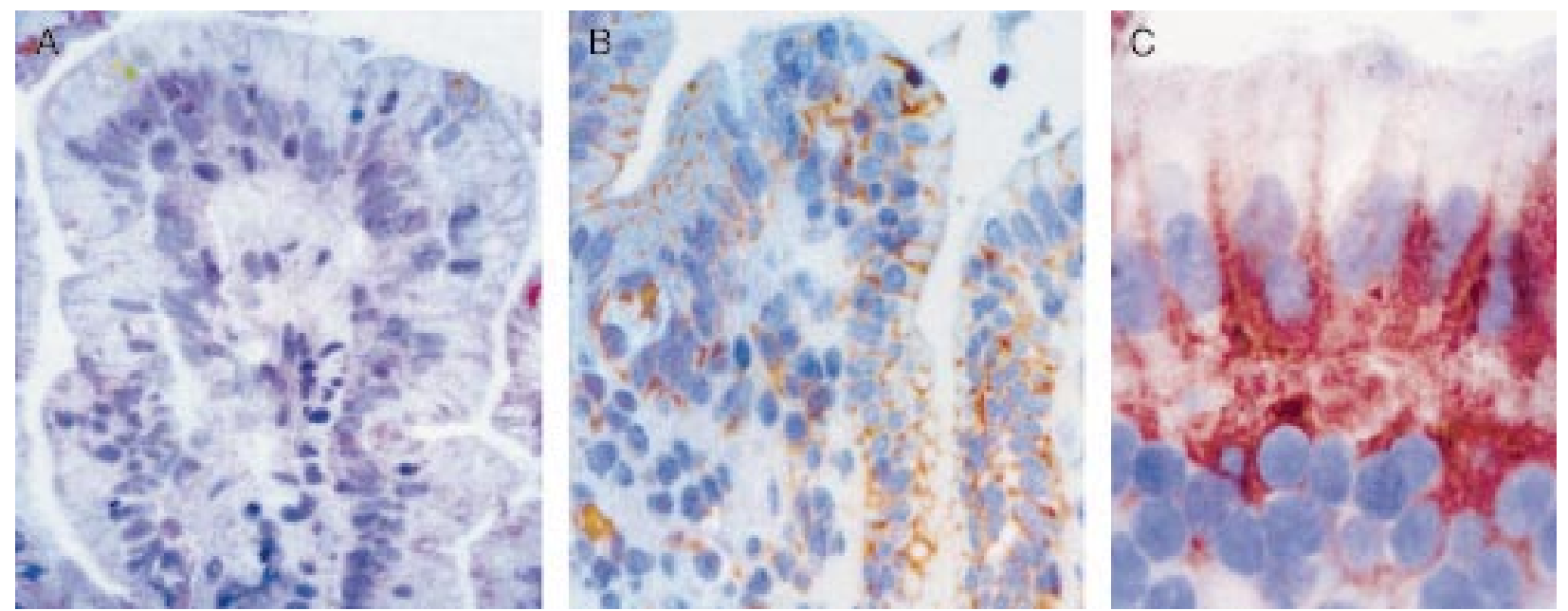

Figure 5 In vitro organ culture of treated coeliac disease (CD) intestine: effect of 24 hour gliadin challenge on enterocyte expression of FAS. (A) FAS expression after 24 hour challenge with medium alone. Faint staining is observed on cell membranes of some enterocytes. (B) FAS expression after 24 hour challenge with gliadin. Intense FAS expression is evident on the cell membranes of the majority of enterocytes and in some lamina propria mononuclear cells. (C) FAS expression after 24 hour challenge with gliadin: high magnification. Staining is evident on the basolateral membranes of the enterocytes. Original magnification: $A, B(\times 160) ; C(\times 240)$; immunohistochemistry, peroxidase staining technique. 
With regard to the pathogenic factors controlling induction of the epithelial phase, the "flat" patches presented with clear signs of epithelial apoptosis and proliferation, as observed by others, ${ }^{37}$ high expression of FAS, evidence of in situ immunological activation, ${ }^{5}$ and spontaneous release of EMA. ${ }^{11}{ }^{20}$ Analysis of patches with a "normal" structure indicated that there were no signs of epithelial involvement (proliferation, apoptosis, or FAS expression) while immunological activation and EMA production were detected in untreated CD patients. ${ }^{1120}$ Thus we have an apparent lack of connection between immunological activation and mucosal damage. Thus these results suggest that the epithelial phase of CD has an apparently more complex mechanism of induction and that it might not be driven solely by immunological recognition of gliadin. Expression of FAS seems to characterise the "flat" regions of the patches and more importantly, FAS engagement has a key role in induction of mucosal damage driven by gliadin challenge. The study using neutralising or agonist anti-FAS antibodies unequivocally indicated that gliadin induced FAS modulation and its engagement has a pivotal role in epithelial damage. Even in this situation it appears that there is a lack of connection between immunorecognition of gliadin and epithelial damage, as we indicated under other experimental conditions. ${ }^{9}$ Indeed, CTLA4-Ig, an extremely efficient device to control $\mathrm{T}$ cell activation, ${ }^{19}$ could not control induction of apoptosis by gliadin in untreated CD explants.

An intriguing finding was that in untreated $\mathrm{CD}$, duodenum regions of "normal" mucosa were contiguous with frank "flat" mucosa and this should spur the quest for an understanding of the mechanisms underlying coeliac lesion. It is difficult to understand why gluten ingestion in these patients causes an uneven pattern of mucosal modification. Our data, demonstrating the presence of signs of local immune activation, indicate that immunological activation may not always lead to mucosal damage. ${ }^{5}$ Most remarkably, even fragments with normal histology produced detectable levels of EMA, indicating disease specific immune activation. These data suggest that EMA by themselves do not induce the histological modification observed in canonical flat mucosa, as recently suggested by Biagi and colleagues, ${ }^{22}$ although we cannot exclude the fact that EMA in combination with other factors may contribute to disease manifestation.

Our study also indicates that there is a hierarchical structure in even the epithelial element as reduction of apoptosis did not lead to a decrease in Ki67 expression. Thus it appears that the proliferative phase is not a consequence of induction of death but rather that proliferation precedes apoptosis. Elucidation of the factors controlling expression of FAS/ apoptosis and induction of $\mathrm{Ki} 67$ could shed further light on the evolution of this pathology. Study of these patchy lesions may be the ideal tool to define the difference between immunological activation and mucosal damage/ remodelling, as immunological activation is a constant feature of untreated CD while mucosal damage/remodelling is not. The financial support of Telethon-Italy (Grant No E762), the
Wellcome Trust (UK), Associazione Italiana Celiachia (Italy) Wellcome Trust (UK), Associazione Italiana Celiachia (Italy) European Community grants QLK1-CT-1999-00037, and
BMH4-98-3703 is gratefully acknowledged. The Kennedy BMH4-98-3703 is gratefully acknowledged. The Kennedy
Institute of Rheumatology is supported by the Arthritis and Institute of Rheumatology
Rheumatism Council UK.

1 Przemioslo RT, Lundin KE, Sollid LM, et al. Histological changes in small bowel mucosa induced by gliadin sensitive T lymphocytes can be blocked by anti-interferon gamma antibody. Gut 1995;36:874-9.

2 Marsh MN. Gluten, major histocompatibility complex, and the small intestine. A molecular and immunobiologic approach to the spectrum of gluten sensitivity ('celiac approach to the spectrum of gluten sen
sprue'). Gastroenterology 1992;102:330-54.

3 Savidge TC, Shmakov AN, Walker-Smith JA, et al. Epithelial cell proliferation in childhood enteropathies. Gut 1996; 39:185-93.

4 MacDonald TT. Epithelial proliferation in response to gastrointestinal inflammation. Ann NY Acad Sci 1992;664: 202-9

5 Picarelli A, Maiuri L, Mazzilli MC, et al. Gluten-sensitive disease with mild enteropathy. Gastroenterology 1996;111: 608-16.

6 Loft DE, Nwokolo CU, Ciclitira PJ. The diagnosis of gluten sensitivity and coeliac disease-the two are not mutually inclusive. Eur $\mathcal{F}$ Gastroenterol Hepatol 1998;10:911-13.

7 Moss SF, Attia L, Scholes JV, et al. Increased small intestine apoptosis in coeliac disease. Gut 1996;39:811-17.

8 Nagata S, Golstein P. The Fas death factor. Science 1995;267:1449-56.

9 Maiuri L, Auricchio S, Coletta S, et al. Blockage of T-cell costimulation inhibits T-cell action in celiac disease. Gastimulation inhibits T-cell action

10 Maiuri L, Picarelli A, Boirivant M, et al. Definition of the initial immunologic modifications upon in vitro gliadin challenge in the small intestine of celiac patients. Gastroenterology 1996;110:1368-78

11 Picarelli A, Maiuri L, Frate A, et al. Production of antiendomysial antibodies after in-vitro gliadin challenge of small intestine biopsy samples from patients with coeliac disease. Lancet 1996;348:1065-7.

12 Auricchio S, De Ritis G, De Vincenzi M, et al. Amines protect in vitro the celiac small intestine from the damaging activity of gliadin peptides. Gastroenterology 1990;99:166874.

13 Auricchio S, De Ritis G, De Vincenzi M, et al. Mannan and oligomers of $\mathrm{N}$-acetylglucosamine protect intestinal mucosa of celiac patients with active disease from in vitro toxcosa of celiac patients with active disease from in vitro tox-
icity of gliadin peptides. Gastroenterology 1990;99:973-8.

14 Alderson MR, Tough TW, Braddy S, et al. Regulation of apoptosis and $\mathrm{T}$ cell activation by Fas-specific mAb. Int apoptosis and T cell activatio

15 Maiuri L, Rossi M, Raia V, et al. Mosaic regulation of lactase in human adult-type hypolactasia. Gastroenterology 1994; 107:54-60

16 Maiuri L, Raia V, De Marco G, et al. DNA fragmentation is a feature of cystic fibrosis epithelial cells: a disease with inappropriate apoptosis? FESEB Lett 1997;408:225-31.

7 Kuitunen P, Kosnai I, Savilahti E. Morphometric study of the jejunal mucosa in various childhood enteropathies with special reference to intraepithelial lymphocytes. $\mathcal{F}$ Pediatr Gastroenterol Nutr 1982;1:525-31.

18 Viard I, Wehrli P, Bullani R, et al. Inhibition of toxic epidermal necrolysis by blockade of CD95 with human intravenous immunoglobulin. Science 1998;282:490-3.

19 Linsley PS, Wallace PM, Johnson J, et al. Immunosuppression in vivo by a soluble form of the CTLA-4 T cell activasion in vivo by a soluble form of the CT
tion molecule. Science 1992;257:792-5.

20 Vogelsang $\mathrm{H}$, Schwarzenhofer M, Granditsch G, et al. In vitro production of endomysial antibodies in cultured duodenal mucosa from patients with celiac disease. Am $\mathcal{F}$ Gastroenterol 1999;94:1057-61

21 Sollid LM, Thorsby E. HLA susceptibility genes in celiac disease: genetic mapping and role in pathogenesis. Gastroenterology 1993;105:910-22

22 Biagi F, Parnell ND, Ellis HJ, et al. Endomysial antibody production is not related to histological damage after in vitro gluten challenge. Eur $\mathcal{F}$ Gastroenterol Hepatol 2000;12: $57-60$. 